\title{
Paramagnetic adsorbates on graphene: A charge transfer analysis
}

\author{
O. Leenaerts, ${ }^{\text {a) }}$ B. Partoens, ${ }^{\text {b) }}$ and F. M. Peeters ${ }^{\text {c) }}$ \\ Departement Fysica, Universiteit Antwerpen, Groenenborgerlaan 171, B-2020 Antwerpen, Belgium
}

(Received 29 May 2008; accepted 3 June 2008; published online 20 June 2008)

\begin{abstract}
We introduce a modified version of the Hirshfeld charge analysis method and demonstrate its accurateness by calculating the charge transfer between the paramagnetic molecule $\mathrm{NO}_{2}$ and graphene. The charge transfer between paramagnetic molecules and a graphene layer as calculated with $a b$ initio methods can crucially depend on the size of the supercell used in the calculation. This has important consequences for adsorption studies involving paramagnetic molecules such as $\mathrm{NO}_{2}$ physisorbed on graphene or on carbon nanotubes. (C) 2008 American Institute of Physics.
\end{abstract}

[DOI: 10.1063/1.2949753]

The use of carbon nanotubes ${ }^{1-6}$ and more recently graphene ${ }^{7}$ as very sensitive gas sensors has stimulated a lot of theoretical work on this subject. ${ }^{8-15}$ Through $a b$ initio calculations, one investigated the adsorption properties of these carbon materials and looked for the mechanisms behind their good sensing capabilities. It appears that the key issue is the charge transfer from the gas molecules to the carbon surface. Such $a b$ initio calculations were able to provide good qualitative agreement with experiment, e.g., whether the gas molecules act as electron donors or acceptors, but a large variation in the size of the doping was found between different theoretical calculations. For example, the calculated charge transfer between a $(10,0)$ single-walled carbon nanotube (SWCNT) and a $\mathrm{NO}_{2}$ molecule varies from ${ }^{9}-0.015 e$ to ${ }^{10}$ $-0.10 e$, an order of magnitude difference. The size of the doping is, however, a crucial factor determining the sensitivity of the gas sensor. In this letter we demonstrate that important reasons for the discrepancies are (i) the different sizes of the supercells used in these $a b$ initio calculations and (ii) the theoretical charge analysis method that has been used. We will show that the former is a very decisive parameter when the adsorbing molecules are paramagnetic. First we examine critically different theoretical charge analysis methods. Then we will perform $a b$ initio calculations with different supercells to investigate the charge transfer dependence on the size of the supercell.

All our $a b$ initio calculations were done within the density funcional theory (DFT) formalism using the ABINIT (Ref. 16) software package within the local spin density approximation and with Troullier-Martins pseudopotentials. ${ }^{17} \mathrm{We}$ used a plane wave basis set with an energy cutoff of $816 \mathrm{eV}$, which was tested to give converged results for all the properties studied in this letter. Different graphene supercells ranging from $2 \times 2$ to $6 \times 6$ were implemented. For the sampling of the Brillouin zone (BZ) we used Monckhorts-Pack (MP) grids for the different supercells equivalent to a range from $12 \times 12 \times 1$ to $48 \times 48 \times 1$ points for a single unit cell.

To calculate the charge transfer from a molecule to a surface, one needs a physically meaningful and transparent approach to divide the electron density between them. A variety of methods has been developed for this purpose, such as the Mulliken's ${ }^{18}$ or Bader's ${ }^{19}$ atoms in molecule approaches. We introduce a method based on the Hirshfeld ${ }^{20}$ method and,

\footnotetext{
${ }^{a}$ Electronic mail: ortwin.leenaerts@ua.ac.be.

b)Electronic mail: bart.partoens@ua.ac.be.

${ }^{c)}$ Electronic mail: francois.peeters@ua.ac.be.
}

in particular, the recently extended version of this approach, the Hirshfeld-I (Ref. 21) method. The simplicity and low cost of computation time makes the Hirshfeld method a very powerfull approach in DFT calculations, and we will demonstrate that its extended version is more accurate than the Bader method and other Hirshfeld-based methods. In the Hirshfeld charge analysis, the total electron density $\rho$ is divided between the different atoms of a system, according to the density of the neutral atoms, $\rho_{A}^{0}$, in free space which build up this system: $Q_{A}=\int\left[\rho_{A}^{0}(\mathbf{r}) / \Sigma_{A^{\prime}} \rho_{A^{\prime}}^{0}(\mathbf{r})\right] \rho(\mathbf{r}) d \mathbf{r}$, with $Q_{A}$ as the charge on atom $A$ (in units of $e$ ). The Hirshfeld-I method does not use the density of neutral atoms in free space, but uses the density of charged atoms instead. These charges on the different atoms are determined through an iterative process. One starts with the simple Hirshfeld method and determines the charge per atom. Then this charge is placed on the different atoms in free space and their densities are used to divide the density again among the atoms as with the simple Hirshfeld method. This procedure is repeated until the charges on the atoms are converged. In our approach we allow our system to relax completely and calculate the total density. Then we calculate the density of the molecule and the graphene layer separately in the same configuration as in the total relaxed system. These densities can now be used in the Hirshfeld-I method instead of the separate atomic densities. Such an approach gives charge transfers that are more physically meaningful as we will demonstrate in the following.

To test this charge analysis procedure we investigate the adsorption of the paramagnetic $\mathrm{NO}_{2}$ molecule (with magnetic moment $M=1 \mu_{b}$ ) on a $4 \times 4$ graphene supercell. We calculate the charge transfer of the $\mathrm{NO}_{2}$-graphene system using different theoretical approaches: we first use the simple Hirshfeld method, then we change the atomic densities into a molecular and a graphene density and use the same procedure; finally we put charges on $\mathrm{NO}_{2}$ and graphene in order to use them in the Hirshfeld-I charge analysis. The Hirshfeld methods that use molecular densities instead of atomic densities will be referred to as the modified Hirshfeld and modified Hirshfeld-I method. For comparison, we also calculated the charge transfer based on the Bader charge analysis. The charge transfer results are given in Table I.

The advantage of using a paramagnetic molecule to demonstrate the accurateness of this charge transfer analysis method is that we can have a good estimate of the charge on the molecule through some physically relevant properties of the system, such as the magnetic moment: extra charge in the 
TABLE I. The charge transfer from graphene to $\mathrm{NO}_{2}$ calculated with different methods.

\begin{tabular}{lc}
\hline \hline \multicolumn{1}{c}{ Method } & Charge transfer $(e)$ \\
\hline Hirshfeld & -0.099 \\
Modified Hirshfeld & -0.161 \\
Modified Hirshfeld-I & -0.181 \\
Bader & -0.212 \\
Magnetic moment & -0.182 \\
\hline \hline
\end{tabular}

partially occupied molecular orbital (POMO) of $\mathrm{NO}_{2}$ will lower the magnetic moment of the $\mathrm{NO}_{2}$ molecule. Extra charge on the graphene layer, on the other hand, will not effect its magnetic moment because pure graphene is diamagnetic. ${ }^{22}$ This implies that we can simply use the lowering of the magnetic moment of the total system (i.e., the difference from $1 \mu_{b}$ ) to estimate the charge transfer between the $\mathrm{NO}_{2}$ molecule and graphene. This is very accurate if there is not too much hybridization between the molecular orbitals of $\mathrm{NO}_{2}$ and the graphene orbitals. This is indeed the case for $\mathrm{NO}_{2}$ since it physisorbs on graphene. ${ }^{13,14}$ The value of the charge transfer extracted from the lowering of the magnetic moment is $-0.182 e$ (see Table I), which is very close to the charge transfer obtained from the modified Hirshfeld-I method. The use of molecular densities instead of atomic ones leads already to a significant improvement of the simple Hirshfeld method and the iterative procedure makes the calculated charge transfer almost equal to the one extracted from the change in magnetic moment of the total system. The small difference of the order of 0.001e is caused by a small charge transfer due to orbital hybridization. This causes no change in the magnetic moment, but it is noticeable in the modified Hirshfeld-I charge analysis. Note also that the charge transfer from the Bader analysis does not correspond with the charge transfer from the lowering of the magnetic moment (see Table I). Thus the modified Hirshfeld-I method gives a good approximation to the charge transfer in the adsorption process of paramagnetic molecules on graphene and, if the molecules are not paramagnetic, it is probably also more accurate to use this modified Hirshfeld-I charge analysis instead of, e.g., the Bader charge analysis for a trustworthy determination of the charge transfer.

Another element of support for the modified Hirshfeld-I method is its geometrical interpretation. When we place an extra charge on a freestanding paramagnetic molecule, it will be placed at the POMO. In the case of a $\mathrm{NO}_{2}$ molecule, this is an antibonding orbital which means that the bond length between the $\mathrm{N}$ and $\mathrm{O}$ atoms becomes larger when the POMO becomes filled. This fact can be used to estimate the charge transfer of a physisorbed $\mathrm{NO}_{2}$ molecule to graphene by comparing the bond length of the relaxed physisorbed molecule with a charged free standing $\mathrm{NO}_{2}$ molecule (see Fig. 1). From Fig. 1 we extract a charge transfer of $-0.206 e$, which is close to the charge transfer obtained by the modified Hirshfeld-I method (and not close to the simple and modified Hirshfeld methods).

Next we will discuss how the charge transfer for the case of paramagnetic molecules depends on the size of the supercell used in the $a b$ initio calculations. The simulation of adsorption processes at surfaces is necessarily restricted to finite supercells. In most cases this gives a reasonably good approximation for, e.g., charge transfer analyses in physDownloaded 23 Jun 2008 to 143.129.131.61. Redistribution subject

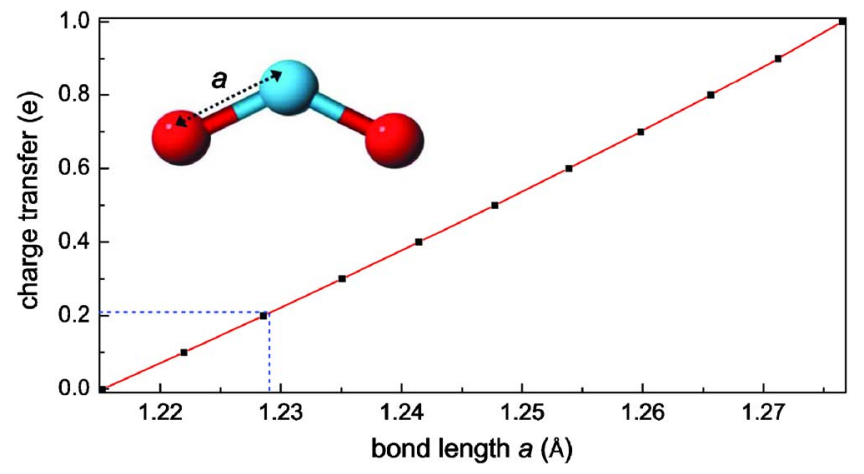

FIG. 1. (Color online) The dependence of the bond length of a $\mathrm{NO}_{2}$ molecule on the extra charge placed on the molecule.

isorption processes, but in some cases, when e.g., the adsorbates are paramagnetic, a different kind of charge transfer mechanism dominates the charge transfer, ${ }^{13,14}$ leading to very different results. We will use the $\mathrm{NO}_{2}$ molecule again to show how sensitive the charge transfer can be with respect to the size of the used supercell. We make use of four different supercells, $2 \times 2,3 \times 3,4 \times 4$, and $6 \times 6$ and several MP grids for sampling the BZ. Figure 2 shows that the charge transfers are converged reasonably well for a $24 \times 24 \times 1$ MP grid.

There is a pronounced dependence of the charge transfer on the number of atoms in the simulated graphene layer ${ }^{23}$ which can be explained by looking at the density of states (DOS) of the total system ${ }^{13,14}$ (see Fig. 3). The lowest unoccupied molecular orbital (LUMO) of $\mathrm{NO}_{2}$ is below the Dirac point $^{24}$ of graphene, which causes a charge transfer to the molecule. This charge transfer depends clearly on the number of electronic states between the Dirac point and the LUMO of the $\mathrm{NO}_{2}$ molecule, which depends linearly on the number of carbon atoms in the supercell. Therefore, we may expect that if one takes a graphene supercell that is large enough, one would eventually get a charge transfer of one electron. ${ }^{13}$ However, it is clear from Fig. 2 that there is no simple linear dependence. The position of the LUMO in the DOS depends on the filling of this orbital: the more electrons in the orbital the more difficult it gets to place another one in

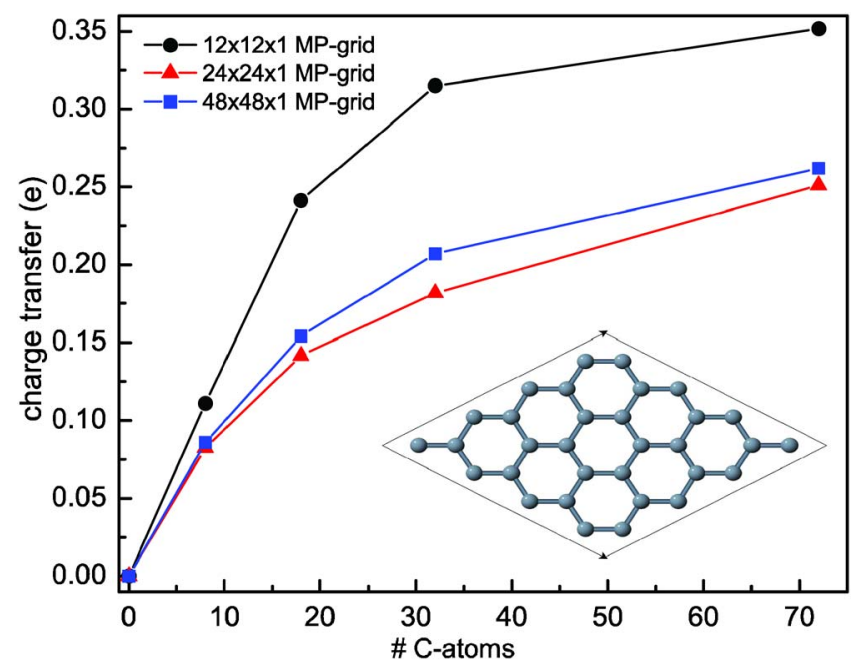

FIG. 2. (Color online) Supercell dependence of the charge transfer between a $\mathrm{NO}_{2}$ molecule and graphene for different MP grids. The inset shows a 4 $\times 4$ graphene supercell. 


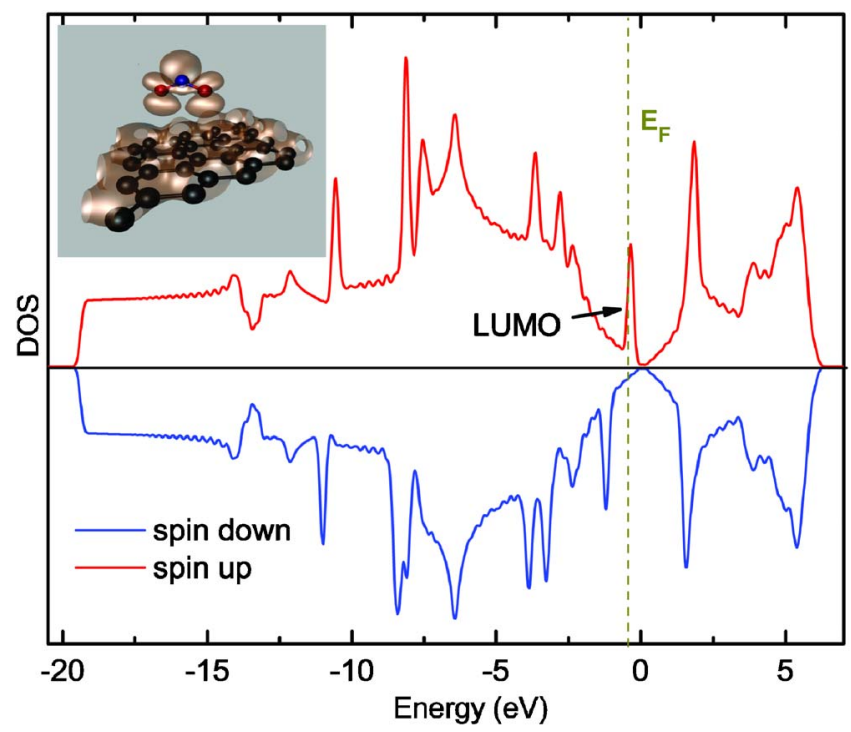

FIG. 3. (Color online) Spin-polarized DOS for $\mathrm{NO}_{2}$ adsorbed on graphene calculated with a $60 \times 60 \times 1 \mathrm{MP}$ grid for a $4 \times 4$ supercell. The LUMO of the $\mathrm{NO}_{2}$ molecule and the Fermi energy are indicated. The inset shows the orbitals of the $\mathrm{NO}_{2}$ molecule (the LUMO) and graphene between which the electron transfer takes place.

it and this translates in a shift of the LUMO toward the Dirac point. In Fig. 4 we show this shift as a function of the number of carbon atoms in the supercell. It is not obvious that the orbital becomes totally filled (a transfer of one electron per paramagnetic molecule) before it coincides with the Dirac point, where the shift will stop and the charge transfer will be converged at a value of less than one electron per molecule.

Thus one can only obtain a quantitative meaningful value of the charge transfer between a paramagnetic molecule and a graphene layer in two cases: (i) when the supercell used is large enough to get a charge transfer of one electron per molecule or (ii) the LUMO of the molecule coincides with the Dirac point and the charge transfer has converged. In the case of the $\mathrm{NO}_{2}$ molecule, one needs a supercell that is much larger than the $6 \times 6$ supercell we were able to use due to computational limitations. However, from Fig.

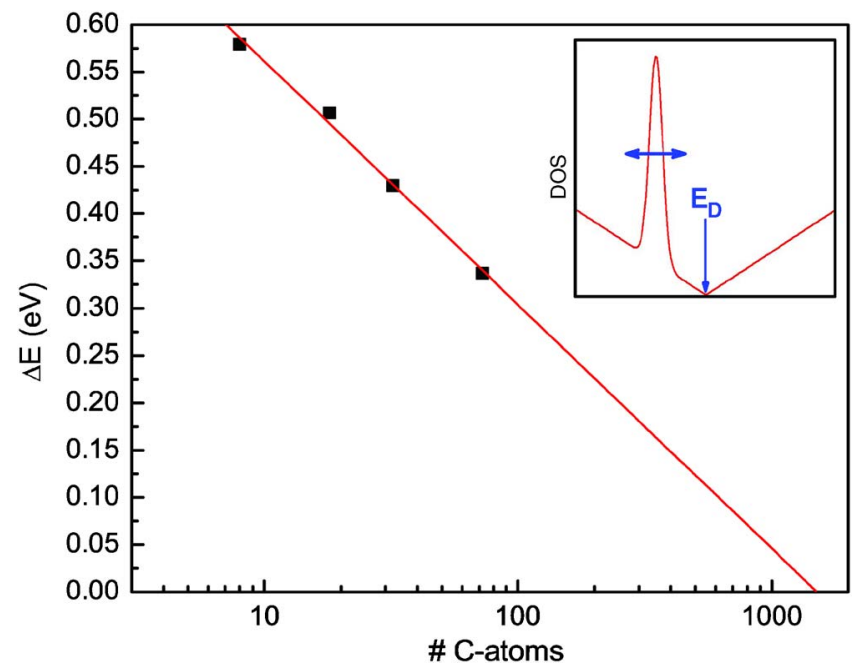

FIG. 4. (Color online) Distance, $\Delta E$, of the $\mathrm{NO}_{2}$ LUMO to the Dirac point with respect to the number of carbon atoms used in the supercell. The inset shows a close up of the total DOS of graphene with an adsorbed $\mathrm{NO}_{2}$ molecule around the Dirac point.
4 we find that if the LUMO stays partially full, it takes more than a thousand carbon atoms to let it coincide with the Dirac point. On the other hand, from Fig. 2 one may deduce that it is not unlikely that a charge transfer of $1 e$ is reached before the supercell contains 1000 carbon atoms. From this we can conclude that probably the LUMO of $\mathrm{NO}_{2}$ will be completely filled before it reaches the Dirac point. This is compatible with the experimental observation ${ }^{7}$ that the charge transfer is equal to one electron charge per $\mathrm{NO}_{2}$ molecule.

To conclude, we examined different methods to calculate the charge transfer between a paramagnetic molecule and a graphene layer and found that a modified Hirshfeld-I method gives the most accurate and physically meaningful results. We used this method to investigate the dependence of the charge transfer on the size of the supercell in ab initio calculations and found that charge transfers involving paramagnetic molecules are, in a nontrivial way, very sensitive to the supercell size. We applied our results to the adsorption of $\mathrm{NO}_{2}$ on graphene and showed that our ab initio calculations are compatible with the experimentally found charge transfer of $1 e .^{7}$ In contrast to the claim in Ref. 13, we found that a charge transfer of $1 e$ is not always realized for adsorption of a paramagnetic molecules on graphene. The present results are also valid for SWCNTs because their adsorption properties are similar to those of graphene. ${ }^{14}$

This work was supported by the Flemish Science Foundation (FWO-Vl), the NOI-BOF of the University of Antwerp, and the Belgian Science Policy (IAP). Discussions with C. Van Alsenoy are gratefully acknowledged.

${ }^{1}$ J. Kong, N. R. Franklin, C. Zhou, M. G. Chapline, S. Peng, K. Cho, and H. Dai, Science 287, 622 (2000).

${ }^{2}$ J. Li, Y. Lu, Q. Ye, M. Cinke, J. Han, and M. Meyyappan, Nano Lett. 3, 929 (2003)

${ }^{3}$ K. Bradley, J.-C. P. Gabriel, M. Briman, A. Star, and G. Grüner, Phys. Rev. Lett. 91, 218301 (2003).

${ }^{4}$ P. Qi, O. Vermesh, M. Grecu, A. Javey, Q. Wang, H. Dai, S. Peng, and K. J. Cho, Nano Lett. 3, 347 (2003).

${ }^{5}$ C. Staii, A. T. Johnson, Jr., M. Chen, and A. Gelperin, Nano Lett. 5, 1774 (2005).

${ }^{6}$ X. Tang, S. Bansaruntip, N. Nakayama, E. Yenilmez, Y.-I. Chang, and Q. Wang, Nano Lett. 6, 1632 (2006).

${ }^{7}$ F. Schedin, A. K. Geim, S. V. Morozov, E. W. Hill, P. Blake, M. I. Katsnelson, and K. S. Novoselov, Nat. Mater. 6, 652 (2007).

${ }^{8}$ S. Peng and K. Cho, Nanotechnology 11, 57 (2000).

${ }^{9}$ S. Santucci, S. Picozzi, F. Di Gregorio, L. Lozzi, C. Cantalini, L. Valentini, J. M. Kenny, and B. Delley, J. Chem. Phys. 119, 10904 (2003).

${ }^{10}$ S. Peng, K. Cho, P. Qi, and H. Dai, Chem. Phys. Lett. 387, 271 (2004).

${ }^{11}$ J. Zhao, A. Buldum, J. Han, and J. P. Lu, Nanotechnology 13, 195 (2002).

${ }^{12}$ E. H. Hwang, S. Adam, and S. Das Sarma, Phys. Rev. B 76, 195421 (2007).

${ }^{13}$ T. O. Wehling, K. S. Novoselov, S. V. Morozov, E. E. Vdovin, M. I. Katsnelson, A. K. Geim, and A. I. Lichtenstein, Nano Lett. 8, 173 (2008).

${ }^{14}$ O. Leenaerts, B. Partoens, and F. M. Peeters, Phys. Rev. B 77, 125416 (2008).

${ }^{15}$ I. Zanella, S. Guerini, S. B. Fagan, J. M. Filho, and A. G. S. Filho, Phys. Rev. B 77, 073404 (2008).

${ }^{16}$ URL: http://www.abinit.org/

${ }^{17}$ N. Troullier and J. L. Martins, Phys. Rev. B 43, 1993 (1991).

${ }^{18}$ R. S. Mulliken, J. Chem. Phys. 23, 2343 (1955).

${ }^{19}$ R. F. Bader, Chem. Rev. (Washington, D.C.) 91, 893 (1991).

${ }^{20}$ F. L. Hirshfeld, Theor. Chim. Acta 44, 129 (1977).

${ }^{21}$ P. Bultinck, C. Van Alsenoy, P. W. Ayers, and R. Carbó-Dorca, J. Chem. Phys. 126, 144111 (2007).

${ }^{22}$ J. W. McClure, Phys. Rev. 104, 666 (1956).

${ }^{23}$ The number of atoms in the simulated graphene layer is equal to twice the number of unit cells in the supercell.

${ }^{24}$ The Dirac point is the Fermi level of pure graphene. 